\title{
Lithium abundance is a gravitational model dependent quantity
}

\author{
Aneta Wojnar 1, * \\ ${ }^{1}$ Laboratory of Theoretical Physics, Institute of Physics, \\ University of Tartu, W. Ostwaldi 1, 50411 Tartu, Estonia
}

\begin{abstract}
The dependence of lithium abundance on modified gravity in low-mass stellar objects is demonstrated. This may introduce an additional uncertainty to age determination techniques of young stars and globular clusters if they rely on the light element depletion method.
\end{abstract}

\section{INTRODUCTION}

Many alternatives to General Relativity (GR) have been proposed in order to shed light on the dark energy and dark matter problems [1-6], the existence of spacetime singularities 7], and the unification with the high energy physics [8, 9], between others. Issues related to astrophysical objects also contribute to the above list of current shortcomings of GR, among them for example the observations of neutron stars with two solar masses [10 12], of a compact object with mass $2.6 M_{\odot}$ [13] sneaking out the mass bounds given by theoretical models for the heaviest neutron stars and the lightest black holes, and very recently, of a binary black hole merger with a total mass of $150 M_{\odot}$ [14, 15].

Pre-main sequence low-mass stars $\left(M \lesssim 0.5 M_{\odot}\right)$ turn out to be interesting objects to study in the context of modified gravity - it was shown that minimum main sequence mass (MMSM) [16 19], cooling process of brown dwarfs [20], as well as an upper mass' limit of fully convective stars on the Main Sequence and Hayashi tracks 21] can be used to constrain theories of gravity. Moreover, during the Hayashi contraction phase those young stars fuse lithium ${ }^{7} \mathrm{Li}$ which depletes before they reach the Main Sequence - that is, the temperature required for lithium burning is lower than the one needed for hydrogen fusion. Therefore, the lithium line is not present in spectroscopically observed red dwarfs with masses $M \lesssim 0.5 M_{\odot}$ in contrast to brown dwarfs whose core temperatures do not reach $\sim 2.5 \times 10^{6} \mathrm{~K}$ which is required for lithium burning. This fact, called lithium test [22, 23], although not ideal, is used in order to distinguish brown dwarfs from Main Sequence stars in the case when one deals with very low-mass and cool stellar (and substellar) objects occupying overlapping regions of effective temperature and luminosity [24].

The lithium abundance at the photosphere in the premain sequence stars is an age-dependent quantity 2528]. It allows to determine clusters' age in the age range of $20-200 \mathrm{Myr}$, being one of the most reliable methods for young globular clusters' age determination. It also means that the lithium depletion boundary method is usually applied to stars' groups of similar age; however, it may provide limits on the ages of LMS individuals.

\footnotetext{
* aneta.magdalena.wojnar@ut.ee
}

More importantly, the procedure is employed to calibrate other techniques used for age estimation since the method is built on solid physical ground, with very few assumptions. Furthermore, the theoretical ages obtained from the lithium depletion method depend weakly on stellar compositions, which is why they do not provide observational uncertainties 29] related to, for instance, star's metallicity. Together with keeping the effective temperature as a free parameter the technique allows to avoid further uncertainties related to atmosphere and convection models [27, 28].

Another prominent feature of the pre-main sequence low-mass stars, which we are going to use in this paper, is their theoretical description. Being fully convective, they can be modelled as a well-mixed polytrope with $n=3 / 2$ even during the last stages of contraction, when electron degeneracy starts being important [30]. Due to the simplified relations, low-mass stars can be modelled by the non-relativistic hydrostatic equilibrium equation, which turn out to be altered by modified gravity (see 31] and references therein). That fact does not only provide tests for modified gravity as already mentioned, but, what we would like to demonstrate in the following discussion, introduces a new uncertainty to the ages of young stars and globular clusters obtained from the lithium depletion boundary method.

Having this in mind, we are going to demonstrate in this work that the lithium abundance in low-mass stars turn out to be dependent on a gravitational model. In the next section [I] we will briefly recall the main features of Palatini $f(\mathcal{R})$ gravity for which we will present the mentioned dependence - however, a similar dependence will also appear in any other model of gravity which alters the stellar description in Newtonian limit. The section III will provide us the main steps of the derivation of light elements abundance in Palatini gravity and we will examine central temperatures, ages and luminosities of a young low-mass star with respect to the GR model. In the last section IV] we will conclude our findings.

Let us add that we use the $(-+++)$ metric signature convention while $\kappa=-\frac{8 \pi G}{c^{4}}[32]$.

\section{PALATINI $f(\mathcal{R})$ GRAVITY}

Palatini $f(\mathcal{R})$ gravity is one of the simplest generalization of GR - instead of considering the linear Lagrangian of the Ricci scalar, one deals with its general functional, 
such as:

$$
S=S_{\mathrm{g}}+S_{\mathrm{m}}=\frac{1}{2 \kappa} \int \sqrt{-g} f(\mathcal{R}) d^{4} x+S_{\mathrm{m}}\left[g_{\mu \nu}, \psi_{m}\right],
$$

where $\mathcal{R}=\mathcal{R}^{\mu \nu} g_{\mu \nu}$ is the Ricci scalar. It is however constructed with the metric $g_{\mu \nu}$ and Ricci tensor $\mathcal{R}_{\mu \nu}$ built of the independent connection $\hat{\Gamma}$ since the common assumption on $g$-metricity of $\hat{\Gamma}$ is discarded. The field equations are provided by the variation of (1) with respect to the metric $g_{\mu \nu}$

$$
f^{\prime}(\mathcal{R}) \mathcal{R}_{\mu \nu}-\frac{1}{2} f(\mathcal{R}) g_{\mu \nu}=\kappa T_{\mu \nu},
$$

where $T_{\mu \nu}$ is the energy momentum tensor of the matter field, $T_{\mu \nu}=-\frac{2}{\sqrt{-g}} \frac{\delta S_{m}}{\delta g_{\mu \nu}}$. In the further part of this paper we will assume a perfect fluid form. The prime in (2) denotes the derivative with respect to the function's argument, that is, $f^{\prime}(\mathcal{R})=\frac{d f(\mathcal{R})}{d \mathcal{R}}$. On the other hand, the variation with respect to the independent connection $\hat{\Gamma}$ gives

$$
\hat{\nabla}_{\beta}\left(\sqrt{-g} f^{\prime}(\mathcal{R}) g^{\mu \nu}\right)=0,
$$

which demonstrates that $\hat{\nabla}_{\beta}$ is the covariant derivative calculated with respect to $\hat{\Gamma}$. In other words, it is the Levi-Civita connection of the conformal metric $h_{\mu \nu}$

$$
h_{\mu \nu}=f^{\prime}(\mathcal{R}) g_{\mu \nu} .
$$

The trace equation, obtained by contracting (2) with the metric $g_{\mu \nu}$, is

$$
f^{\prime}(\mathcal{R}) \mathcal{R}-2 f(\mathcal{R})=\kappa T,
$$

where $T$ is the trace of the energy-momentum tensor, and it allows to obtain the relation $\mathcal{R}=\mathcal{R}(T)$ for some chosen functional $f(\mathcal{R})$.

An important feature of the Palatini gravity, which can be derived easily from (5), is that in the vacuum it provides Einstein vacuum solution with the cosmological constant, independently of the form of $f(\mathcal{R})$. Furthermore, in the case of analytic $f(\mathcal{R})$ one deals with the same center-of-mass orbits as in GR [33]. Therein, it was also demonstrated that the modifications of energy and momentum present in Euler equation are not sensitive to the experiments performed so far for the solar system orbits. This may change when atomic level experiments will be available, though [34 36].

We may rewrite the field equations (2) as dynamical equations for the conformal metric $h_{\mu \nu}$ [37 39] and the undynamic scalar field defined as $\Phi=f^{\prime}(\mathcal{R})$ :

$$
\begin{gathered}
\bar{R}_{\mu \nu}-\frac{1}{2} h_{\mu \nu} \bar{R}=\kappa \bar{T}_{\mu \nu}-\frac{1}{2} h_{\mu \nu} \bar{U}(\Phi) \\
\Phi \bar{R}-\left(\Phi^{2} \bar{U}(\Phi)\right)^{\prime}=0
\end{gathered}
$$

where $\bar{U}(\Phi)=\frac{\mathcal{R} \Phi-f(\mathcal{R})}{\Phi^{2}}$ and the energy momentum tensor in the Einstein frame is given by $\bar{T}_{\mu \nu}=\Phi^{-1} T_{\mu \nu}$. Let us notice that prime here denotes the derivative with respect to $\Phi$. Such a representation can significantly simplify given physical problems 40 43.

\section{A. Non-relativistic stars in Palatini gravity}

In what follows, we will consider the quadratic (Starobinski) model

$$
f(\mathcal{R})=\mathcal{R}+\beta \mathcal{R}^{2},
$$

where $\beta$ is the model parameter with the dimension $\left[\mathrm{m}^{2}\right]$, for which it was shown that the non-relativistic Palatini stars can be described by the equations [19, 44]

$$
\begin{aligned}
\frac{d p}{d \tilde{r}} & =-\frac{G m(\tilde{r}) \rho(\tilde{r})}{\Phi(\tilde{r}) \tilde{r}^{2}}, \\
m & =\int_{0}^{\tilde{r}} 4 \pi x^{2} \rho(x) d x,
\end{aligned}
$$

where $\tilde{r}^{2}=\Phi(\tilde{r}) r^{2}$ and $\Phi(\tilde{r}) \equiv f^{\prime}(\mathcal{R})=1+2 \kappa c^{2} \beta \rho(\tilde{r})$. The transformation to the Jordan frame and the Taylor expansion around $\beta=0$ will provide the modified hydrostatic equilibrium equation as

$$
p^{\prime}=-g \rho\left(1+\kappa c^{2} \beta\left[r \rho^{\prime}-3 \rho\right]\right),
$$

where $g=$ const is the surface gravity assumed to be a constant

$$
g \equiv \frac{G m(r)}{r^{2}} \sim \frac{G M}{R^{2}},
$$

where $M=m(R)$ and $R$ is a star's radius. On the other hand, the transformation of the mass function $m(\tilde{r})$ to $m(r)$ is dependent on the energy density which will drop however to zero on the non-relativistic star's surface. Because of that, the derivation of the mass function has a simple form $m^{\prime}(r)=4 \pi r^{2} \rho(r)$ in the Jordan frame, thus we may write

$$
m^{\prime \prime}=8 \pi r \rho+4 \pi r^{2} \rho^{\prime} .
$$

Using this and (11) in (10), one writes

$$
p^{\prime}=-g \rho\left(1+8 \beta \frac{g}{c^{2} r}\right) .
$$

As discussed already in the introductory section, our concern are low-mass stars whose equation of state can be modelled by a simple polytropic relation

$$
p=K \rho^{\gamma},
$$

which together with the hydrostatic equilibrium provides the Palatini Lane-Emden equation [44]:

$$
\frac{1}{\xi} \frac{d^{2}}{d \xi^{2}}\left[\sqrt{\Phi} \xi\left(\theta-\frac{2 \kappa^{2} c^{2} \rho_{c} \alpha}{n+1} \theta^{n+1}\right)\right]=-\frac{\left(\Phi+\frac{1}{2} \xi \frac{d \Phi}{d \xi}\right)^{2}}{\sqrt{\Phi}} \theta^{n},
$$

where $\Phi=1+2 \alpha \theta^{n}$ with $\alpha$ defined as $\alpha=\kappa c^{2} \beta \rho_{c}$, while the dimensionless variables $\theta$ and $\xi$ are given by

$$
\begin{aligned}
r & =r_{c} \xi, \quad \rho=\rho_{c} \theta^{n}, \quad p=p_{c} \theta^{n+1}, \\
r_{c}^{2} & =\frac{(n+1) p_{c}}{4 \pi G \rho_{c}^{2}} .
\end{aligned}
$$


The function $\theta(\xi)$ is the solution of the (modified) LaneEmden equation (15) with respect to the radial coordinate $\xi=r \rho_{c} \sqrt{8 \pi G /\left(2 p_{c}\right)}$ which crosses zero at $\xi_{R}$. Here, $p_{c}$ and $\rho_{c}$ denote the central pressure and density, respectively, while $n=\frac{1}{\gamma-1}$ is the polytropic index of (14). More detailed discussion about Palatini LaneEmden equation, its solutions, and features can be found in 21, 44 46].

The solution of the modified Lane-Emden equation (15) provide the star's mass, radius, central density, and temperature via the well-known expressions (see e.g [32])

$$
\begin{aligned}
M & =4 \pi r_{c}^{3} \rho_{c} \omega_{n}, \\
R & =\gamma_{n}\left(\frac{K}{G}\right)^{\frac{n}{3-n}} M^{\frac{n-1}{n-3}}, \\
\rho_{c} & =\delta_{n}\left(\frac{3 M}{4 \pi R^{3}}\right), \\
T & =\frac{K \mu}{k_{B}} \rho_{c}^{\frac{1}{n}} \theta_{n},
\end{aligned}
$$

where $k_{B}$ denotes the Boltzmann constant, $\mu$ the mean molecular weight while $K$ contains information about gas mixture and degeneracy of the stellar material. The constants (22) and (24) depend on the central energy density via $\Phi$ and its derivation with respect to $\xi$, which is a common feature of Palatini theories of gravity:

$$
\begin{aligned}
\omega_{n} & =-\left.\frac{\xi^{2} \Phi^{\frac{3}{2}}}{1+\frac{1}{2} \xi \frac{\Phi_{\xi}}{\Phi}} \frac{d \theta}{d \xi}\right|_{\xi=\xi_{R}}, \\
\gamma_{n} & =(4 \pi)^{\frac{1}{n-3}}(n+1)^{\frac{n}{3-n}} \omega_{n}^{\frac{n-1}{3-n}} \xi_{R}, \\
\delta_{n} & =-\frac{\xi_{R}}{\left.3 \frac{\Phi^{-\frac{1}{2}}}{1+\frac{1}{2} \xi \frac{\Phi_{\xi}}{\Phi}} \frac{d \theta}{d \xi}\right|_{\xi=\xi_{R}}} .
\end{aligned}
$$

In the next section we will use the above relations to calculate the lithium depletion rate (which can be also easily generalized for resonant rates).

\section{LITHIUM BURNING IN LOW-MASS MAIN SEQUENCE STARS}

As already discussed, fully convective low-mass stars are very-well described by the formalism given in the subsection (IIA). In such stars, the lithium-to-hydrogen ratio $f$ changes due to the effective convection which mixes lithium-poor and lithium-rich regions throughout a star such that the mixing timescale is much shorter than the contraction and lithium destruction times (that is, the star is well mixed). Apart from this process, the protoncapture reactions also contribute to the rate of change of $f$. Thus, the depletion rate in a star with mass $M$ and hydrogen fraction $X$ can be written in the following way

$$
M \frac{\mathrm{d} f}{\mathrm{~d} t}=-\frac{X f}{m_{H}} \int_{0}^{M} \rho\langle\sigma v\rangle d M
$$

where the non-resonant reaction rate for the temperature range $T<6 \times 10^{6} \mathrm{~K}$ is given by

$$
N_{A}\langle\sigma v\rangle=S f_{\mathrm{scr}} T_{c 6}^{-2 / 3} \exp \left[-a T_{c 6}^{-\frac{1}{3}}\right] \frac{\mathrm{cm}^{3}}{\mathrm{sg}},
$$

where $T_{c 6} \equiv T_{c} / 10^{6} \mathrm{~K}$ and $f_{\text {scr }}$ is the screening correction factor while $S$ and $a$ are dimensionless parameters in the fit to the reaction rate. For our range of temperatures, the proton-capture rate parameters for the reaction ${ }^{7} \mathrm{Li}(p, \alpha){ }^{4} \mathrm{He}$ are $S=7.2 \times 10^{10}$ and $a=84.72$ [28, 47, 48].

Since we are dealing with polytropic stars with the polytropic index $n=3 / 2$, the temperature is $T=T_{c} \theta(\xi)$ while density is expressed as $\rho=\rho_{c} \theta^{3 / 2}(\xi)$. Therefore, the central temperature $T_{c}$ and central density $\rho_{c}$ for that model are modified (via $\delta, \xi_{R}$ and $\theta^{\prime}$ ) and given by

$$
T_{c}=1.15 \times 10^{6}\left(\frac{\mu_{\mathrm{eff}}}{0.6}\right)\left(\frac{M}{0.1 M_{\odot}}\right)\left(\frac{R_{\odot}}{R}\right) \frac{\delta^{\frac{2}{3}}}{\xi_{R}^{\frac{5}{3}}\left(-\theta^{\prime}\left(\xi_{R}\right)\right)^{\frac{1}{3}}} \mathrm{~K}
$$

$\rho_{c}=0.141\left(\frac{M}{0.1 M_{\odot}}\right)\left(\frac{R_{\odot}}{R}\right)^{3} \delta \frac{\mathrm{g}}{\mathrm{cm}^{3}}$

while the radius, when taking into account an arbitrary degeneracy degree $\eta$ and mean molecular weight $\mu_{\text {eff }}$, is

$$
\frac{R}{R_{\odot}} \approx \frac{7.1 \times 10^{-2} \gamma}{\mu_{\mathrm{eff}} \mu_{e}^{\frac{2}{3}} F_{1 / 2}^{\frac{2}{3}}(\eta)}\left(\frac{0.1 M_{\odot}}{M}\right)^{\frac{1}{3}}
$$

where $F_{n}(\eta)$ is the $n$th order Fermi-Dirac function. Inserting the Lane-Emden temperature, energy density and radius to (25) and changing the variables to the spatial ones we will have

$$
\begin{aligned}
\frac{\mathrm{d}}{\mathrm{d} t} \ln f & =-\frac{4 \pi X}{\xi_{R}^{3}} \frac{\rho_{c}^{2} R^{3}}{M} \frac{S}{N_{A} m_{H}}\left(\frac{u}{a}\right)^{2} \\
& \times \int_{0}^{\xi_{R}} f_{\mathrm{scr}} \xi^{2} \theta^{\frac{7}{3}} \exp \left(-u \theta^{-1 / 3}\right) d \xi \frac{1}{\mathrm{~s}},
\end{aligned}
$$

where $u \equiv a T_{6}^{-1 / 3}$. Approximately, the burning process is restricted to the central region of the star, thus we may apply the near center solution of the modified LaneEmden equation (15) to the depletion rate (30)

$$
\theta(\xi \approx 0) \approx 1-\frac{\xi^{2}}{6} \approx \exp \left(-\frac{\xi^{2}}{6}\right),
$$

which after applying the numerical constants yields

$$
\begin{aligned}
\frac{\mathrm{d}}{\mathrm{d} t} \ln f & =-6.54\left(\frac{X}{0.7}\right)\left(\frac{0.6}{\mu_{\mathrm{eff}}}\right)^{3}\left(\frac{0.1 M_{\odot}}{M}\right)^{2} \\
& \times S f_{\mathrm{scr}} a^{7} u^{-\frac{17}{2}} e^{-u}\left(1+\frac{7}{u}\right)^{-\frac{3}{2}} \xi_{R}^{2}\left(-\theta^{\prime}\left(\xi_{R}\right)\right) .
\end{aligned}
$$

The integration of the above equation requires the knowledge of the dependence of the central temperature parameter $u$ on time. In order to find it, let us consider 
Stefan-Boltzman equation together with the virial theorem after the transformation to the Jordan frame

$$
L=4 \pi R^{2} T_{\text {eff }}^{4}=-\frac{3}{7} \Omega \frac{G M^{2}}{R^{2}} \frac{\mathrm{d} R}{\mathrm{~d} t},
$$

where the factor

$$
\Omega=\left(\frac{\Phi^{\frac{3}{2}}}{1+\frac{1}{2} \xi_{R} \frac{\Phi^{\prime}}{\Phi}}\right)^{-\frac{4}{3}}
$$

appears due to the frame transformation [45]. Therefore, it is straightforward to get the radius and luminosity as functions of time during the contraction phase

$$
\begin{aligned}
\frac{R}{R_{\odot}} & =0.85 \Omega^{\frac{1}{3}}\left(\frac{M}{0.1 M_{\odot}}\right)^{\frac{2}{3}}\left(\frac{3000 \mathrm{~K}}{T_{\mathrm{eff}}}\right)^{\frac{4}{3}}\left(\frac{\mathrm{Myr}}{t}\right)^{\frac{1}{3}} \\
\frac{L}{L_{\odot}} & =5.25 \times 10^{-2} \Omega\left(\frac{M}{0.1 M_{\odot}}\right)^{\frac{4}{3}}\left(\frac{T_{\mathrm{eff}}}{3000 \mathrm{~K}}\right)^{\frac{4}{3}}\left(\frac{\mathrm{Myr}}{t}\right)^{\frac{2}{3}}
\end{aligned}
$$

while the contraction time is given by

$$
\begin{aligned}
t_{\text {cont }} \equiv & -\frac{R}{\mathrm{~d} R / \mathrm{d} t} \approx 841.91\left(\frac{3000 \mathrm{~K}}{T_{\text {eff }}}\right)^{4}\left(\frac{0.1 M_{\odot}}{M}\right) \\
& \times\left(\frac{0.6}{\mu_{\text {eff }}}\right)^{3}\left(\frac{T_{c}}{3 \times 10^{6} \mathrm{~K}}\right)^{3} \frac{\xi_{R}^{2}\left(-\theta^{\prime}\left(\xi_{R}\right)\right) \Omega}{\delta^{2}} \mathrm{Myr} .
\end{aligned}
$$

From the relation (29) and (35) we may also write down the degeneracy parameter as a function of time

$$
\mu_{\mathrm{eff}} F_{1 / 2}^{\frac{2}{3}}(\eta) \approx 8.36 \times 10^{-2} \frac{\gamma}{\Omega^{1 / 3}}\left(\frac{0.1 M_{\odot}}{M}\right)\left(\frac{T_{3 \mathrm{eff}}^{4} t_{6}}{\mu_{e}^{2}}\right)^{1 / 3}
$$

where $T_{3 \mathrm{eff}} \equiv T_{\text {eff }} / 3000 \mathrm{~K}$ and $t_{6} \equiv t / 10^{6}$. Then, using (27) together with (29), (35), and (38) we find

$$
\begin{aligned}
\frac{u}{a} & =1.15\left(\frac{M}{0.1 M_{\odot}}\right)^{2 / 9}\left(\frac{\mu_{e} F_{1 / 2}(\eta)}{t_{6} T_{3 e f f}^{4}}\right)^{2 / 9} \\
& \times\left(\frac{\xi_{R}^{5} \Omega^{2 / 3}\left(-\theta^{\prime}\left(\xi_{R}\right)\right)^{2 / 3}}{\gamma \delta^{2 / 3}}\right)^{1 / 3},
\end{aligned}
$$

which relates the central temperature $T_{c}$ with the time during the contraction phase.

Let us consider the case $M \gtrsim 0.2 M_{\odot}$, that is, when the degeneracy effects are not important and $\dot{\mu}_{\text {eff }}$ can be neglected when compared to $\dot{R}$. Then, since $u=a T_{c 6}^{-1 / 3}$ and using (27) will provide $\mathrm{d} u / \mathrm{d} R=u /(3 R)$, such that

$$
\frac{\mathrm{d}}{\mathrm{d} t} \ln f \approx \frac{\mathrm{d} \ln f}{\mathrm{~d} u} \frac{\partial u}{\partial R} \dot{R}=\frac{\mathrm{d} \ln f}{\mathrm{~d} u} \frac{u \dot{R}}{3 R}
$$

which allows to write the depletion rate as

$$
\begin{aligned}
& \frac{\mathrm{d} \ln f}{\mathrm{~d} u}=1.15 \times 10^{13} T_{3 \mathrm{eff}}^{-4}\left(\frac{X}{0.7}\right)\left(\frac{0.6}{\mu_{\mathrm{eff}}}\right)^{6}\left(\frac{M_{\odot}}{M}\right)^{3} \\
& \times S f_{\mathrm{scr}} a^{16} u^{-\frac{37}{2}} e^{-u}\left(1-\frac{21}{2 u}\right) \frac{\xi_{R}^{4}\left(-\theta^{\prime}\left(\xi_{R}\right)\right)^{2} \Omega}{\delta^{2}} .
\end{aligned}
$$

Integrating the above equation from $u_{0}=\infty$ to $u$ and using the properties of the incomplete gamma function gives

$$
\begin{aligned}
\mathcal{F} \equiv \ln \frac{f_{0}}{f} & =1.15 \times 10^{13} T_{3 \mathrm{eff}}^{-4}\left(\frac{X}{0.7}\right)\left(\frac{0.6}{\mu_{\mathrm{eff}}}\right)^{6}\left(\frac{M_{\odot}}{M}\right)^{3} \\
& \times S f_{\mathrm{scr}} a^{16} g(u) \frac{\xi_{R}^{4}\left(-\theta^{\prime}\left(\xi_{R}\right)\right)^{2} \Omega}{\delta^{2}}
\end{aligned}
$$

where $g(u)=u^{-37 / 2} e^{-u}-29 \Gamma(-37 / 2, u)$ with the function $\Gamma(-37 / 2, u)$ being an upper incomplete gamma function. For a given depletion $\mathcal{F}$, the central temperature $T_{c}$ is obtained from $u(\mathcal{F})$ while the star's age, radius, and luminosity are specified by the equations (37), (35), and (36), respectively. Each of those, as demonstrated, is altered by $\Omega, \gamma, \delta, \xi_{R}, \theta\left(\xi_{R}\right)$, and $\theta^{\prime}\left(\xi_{R}\right)$ whose values depend on the applied model of gravity.

Using the similar approach, one may write down the depletion equation (42) for resonant rates (see e.g ([28])), where $j=2 / 3$ stands for the non-resonant reactions,

$$
\begin{aligned}
\mathcal{F} \equiv \ln \frac{f_{0}}{f} & =1.15 \times 10^{13} T_{3 \mathrm{eff}}^{-4}\left(\frac{X}{0.7}\right)\left(\frac{0.6}{\mu_{\mathrm{eff}}}\right)^{6}\left(\frac{M_{\odot}}{M}\right)^{3} \\
& \times S f_{\mathrm{scr}} a^{18-3 j} g(u) \frac{\xi_{R}^{4}\left(-\theta^{\prime}\left(\xi_{R}\right)\right)^{2} \Omega}{\delta^{2}}
\end{aligned}
$$

with $g(u)=u^{-41 / 2-3 j} e^{-u}-\frac{68-15 j}{2} \Gamma\left(-\frac{41}{2}-3 j, u\right)$.

The equations (42) and (43) can be solved numerically or be fitted to the observational data; however, we may also find an approximate formula for the central temperature at the time of depletion. Thus, we will compare the local nuclear destruction time at the center of the star $(X=0.7$ being the hydrogen mass fraction while $m_{p} \approx 1.67 \times 10^{-24} \mathrm{~g}$ is the proton mass)

$$
\begin{aligned}
t_{\mathrm{dest}} & =\frac{m_{p}}{X \rho\langle\sigma v\rangle}=4.92 \times 10^{-7}\left(\frac{M}{0.1 M_{\odot}}\right)^{2} \\
& \times\left(\frac{\mu_{\mathrm{eff}}}{0.6}\right)^{3} \frac{T_{c 6}^{-\frac{7}{3}}}{S f_{\mathrm{scr}}} e^{\frac{a}{T_{c 6}^{13}}} \frac{\delta}{\xi_{R}^{5} \theta^{\prime}\left(\xi_{R}\right)} \mathrm{yr}
\end{aligned}
$$

to the contraction time (37). The approximation $t_{\text {cont }}=$ $t_{\text {dest }}$ works well so long as the star can be described by the polytropic equation of state with $n=3 / 2$ (the degeneracy is not important):

$$
\begin{aligned}
\frac{a}{T_{c 6}^{1 / 3}} & =31.78+\ln \left(S f_{\mathrm{scr}}\right)+\ln \left(\frac{\xi_{R}^{7} \theta^{\prime}\left(\xi_{R}\right)^{2} \Omega}{\delta^{3}}\right)-6 \ln \left(\frac{\mu_{\mathrm{eff}}}{0.6}\right) \\
& -3 \ln \left(\frac{M}{0.1 M_{\odot}}\right)-4 \ln \left(\frac{T_{\mathrm{eff}}}{3000 \mathrm{~K}}\right)+\frac{16}{3} \ln T_{c 6} .
\end{aligned}
$$

Let us now consider a star with $T_{\text {eff }}=3500 \mathrm{~K}$, the mass $M=0.5 M_{\odot}$ with $f_{\text {scr }}=1$ (evaluated at the center of the star) and $\mu_{\text {eff }}=0.6$. For the GR values $(\alpha=0)$ of the polytropic solutions ${ }^{7} \mathrm{Li}$ depletes when the central temperature is $T_{c} \approx 2.98 \times 10^{6} \mathrm{~K}$. A few other values of the parameter $\alpha=\kappa c^{2} \beta \rho_{c}$ and their corresponding 
central temperatures, ages, radii, and luminosities are given in the table (I).

The obtained results clearly demonstrate that modified gravity (here Palatini quadratic model) significantly changes the ages and luminosities of lithium depleted premain sequence stars with respect to the GR model. Despite the fact that the values from the table (II) are given by the approximated expression (45), the deviations from the GR model with $\alpha=0$ in (43) are also expected to occur.

\section{CONCLUSIONS}

In this paper we have obtained the lithium-to-hydrogen ratio for the stellar model provided by the Palatini $f(\mathcal{R})$ gravity. Our main result is given by the equations (42), (43), and (45), where the last one's solutions for a few values of the parameter $\alpha$ are presented in the table (I). All those relations contain terms depending on the solution of (modified) Lane-Emden equation, introducing the dependence on gravitational model of interest. Such a dependence will appear in theories of gravity which modify the Newtonian limit of the relativistic hydrostatic equilibrium equation (that is, the Tolman-OppenheimerVolkoff equation).

Although it seems to be worrying that the lithium depletion based techniques for the age estimation depend on the applied model of gravity, it also gives room for gravitational theories whose modifications shorten any phase of the stellar evolution, as provided by the considered Palatini quadratic model. The discovery of a $0.2 M_{\odot}$ white dwarf in the binary system KIC 8145411 [49] which according to the commonly accepted model would have to be older than the Universe [50], is a clear example of the need of different evolutionary scenarios (for a brief discussion on that topic, see [51]). It was shown 52] that white dwarfs are also found in young clusters whose progenitor stars' masses depend crucially on the assumed age of the cluster, which is another argument for being aware of the discussed dependence when the lithium based method is used.

Moreover, staying shorter (longer) in any evolutionary phase has a noticeable effect on the total stars' luminosity which contributes to the galaxy brightness [53] since the galaxy can have more (less) generations of stars with different luminosities than the ones predicted by the GR model.

In addition, the results discussed in this work may also provide a test for gravitational theories: prolonging prominently low-mass stars' lifetimes in comparison to the current widely accepted model would raise doubts on a theory which introduces such effects. Further studies along these lines are currently underway.

\section{ACKNOWLEDGMENTS}

The work is supported by the Regional Development Fund CoE program TK133 "The Dark Side of the Universe."

\begin{tabular}{|c||c|c|c|c|}
\hline$\alpha$ & $T_{c} / 10^{6} \mathrm{~K}$ & $t[\mathrm{Myr}]$ & $R / R_{\odot}$ & $L / L_{\odot}$ \\
\hline \hline-0.4 & 3.48 & 3.21 & 1.85 & $25.3 \times 10^{-2}$ \\
-0.1 & 3.18 & 7.48 & 1.28 & $14.4 \times 10^{-2}$ \\
-0.001 & 3.129 & 7.76 & 1.19 & $14.1 \times 10^{-2}$ \\
\hline $0(\mathrm{GR})$ & 2.98 & 12.42 & 1.03 & $10.3 \times 10^{-2}$ \\
\hline 0.001 & 3.128 & 7.78 & 1.19 & $14 \times 10^{-2}$ \\
0.1 & 3.098 & 7.25 & 1.13 & $14.7 \times 10^{-2}$ \\
0.4 & 3.093 & 3.57 & 1.06 & $23.6 \times 10^{-2}$ \\
\hline
\end{tabular}

Table I. Numerical values of central temperatures (in $10^{6} \mathrm{~K}$ ), age (in Myr), radius (in $R_{\odot}$ ), and luminosity (in $L_{\odot}$ ) of fully convective low-mass stars with respect to $\alpha=\kappa c^{2} \beta \rho_{c}$ at the time of ${ }^{7} \mathrm{Li}$ depletion. The star's mass, effective temperature, hydrogen mass fraction, and mean molecular weight are $M=$ $0.5 M_{\odot}, T_{\text {eff }}=3500 \mathrm{~K}, X=0.7$, and $\mu_{\text {eff }}=0.6$, respectively.
[1] E. J. Copeland, M. Sami, and S. Tsujikawa, Int. J. Mod. Phys. D 15 (2006) 1753.

[2] S. Nojiri and S. D. Odintsov, Int. J. Geom. Meth. Mod. Phys. 4 (2007) 115.

[3] S. Nojiri, S.D. Odintsov, V.K. Oikonomou, Modified Gravity Theories on a Nutshell: Inflation, Bounce and Late-time Evolution, Physics Reports 692 (2017).

[4] S. Nojiri, S.D. Odintsov, Unified cosmic history in modified gravity: from $F(R)$ theory to Lorentz non-invariant models, Physics Reports 505 (2011).

[5] S. Capozziello and M. Francaviglia, Gen. Rel. Grav. 40 (2008) 357.

[6] S. M. Carroll, A. De Felice, V. Duvvuri, D. A. Easson, M. Trodden, and M. S. Turner, Phys. Rev. D 71 (2005) 063513.
[7] J. M. M. Senovilla and D. Garfinkle, Class. Quant. Grav. 32 (2015) 124008.

[8] L. Parker and D. J. Toms, "Quantum Field Theory in Curved Spacetime: Quantized Fields and Gravity" (Cambridge University Press, Cambridge, England, 2009).

[9] N. D. Birrel and P. C. W. Davies, "Quantum Fields in Curved Space" (Cambridge University Press, Cambridge, England, 1982).

[10] M. Linares, T. Shahbaz, and J. Casares, The Astrophysical Journal 859 (2018) 54.

[11] J. Antoniadis et al., Science 340 (2012) 6131.

[12] F. Crawford, M. S. E. Roberts, J. W. T. Hessels, S. M. Ransom, M. Livingstone, C. R. Tam and V. M. Kaspi, Astrophys. J. 652 (2006) 1499.

[13] R. Abbott et al, The Astrophysical Journal 896 L44 
(2020).

[14] R. Abbott et al. (LIGO Scientific Collaboration and Virgo Collaboration), Phys. Rev. Lett. 125, 101102 (2020).

[15] J. Sakstein, et al., arXiv:2009.01213.

[16] J. Sakstein, Phys. Rev. Lett. 115 (2015) 201101.

[17] J. Sakstein, Phys. Rev. D 92 (2015) 124045.

[18] M. Crisostomi, M. Lewandowski and F. Vernizzi, Phys. Rev. D 100 no.2, 024025 (2019).

[19] G.J. Olmo, D. Rubiera-Garcia, A. Wojnar, Phys. Rev. D 100.4 (2019), 044020.

[20] M. Benito, A. Wojnar, arXiv:2101.02146.

[21] A. Wojnar, Phys. Rev. D 102 (2020) 124045.

[22] R. Rebolo, E.L. Martin, A. Magazzu, The Astrophysical Journal 389 (1992): L83-L86.

[23] L.A. Nelson, S. Rappaport, E. Chiang, The Astrophysical Journal 413 (1993): 364-367.

[24] G. Bastri, The Lithium Test for Young Brown Dwarfs (invited review). In: Brown dwarfs and extrasolar planets. 1998. p. 394.

[25] G. Basri, G.W. Marcy, J.R. Graham, The Astrophysical Journal 458 (1996): 600.

[26] G. Chabrier. I. Baraffe, The Astrophysical Journal, 459: L91-L94 (1996).

[27] L. Bildsten, et al., The Astrophysical Journal 482.1 (1997): 442.

[28] G. Ushomirsky et al., The Astrophysical Journal 497.1 (1998): 253.

[29] D.R. Soderblom, et al. Ages of young stars Protostars and Planets VI 3.1 (2014).

[30] A. Burrows and J. Liebert, Rev. Mod. Phys. 65 (1993) 301.

[31] G.J. Olmo, D. Rubiera-Gracia, A. Wojnar, Stellar structure models in modified theories of gravity: lessons and challenges, Physics Reports 876 (2020).

[32] S. Weinberg, Gravitation and Cosmology: principles and Applications of the General Theory of Relativity, John
Wiley and Sons (1972).

[33] Toniato, J.D.; Rodrigues, D.C.; Wojnar, A., Phys. Rev. D 2020, 101, 064050.

[34] P. K. Schwartz and D. Giulini, Phys. Rev. A 100, 052116 (2019).

[35] G. J. Olmo, Phys. Rev. D 77, 084021 (2008).

[36] G. J. Olmo, Phys. Rev. Letters 98, 061101 (2007).

[37] A. De Felice and S. Tsujikawa, Living Rev. Rel. 13 (2010) 3.

[38] A. Stachowski, M. Szydlowski, A. Borowiec, Eur. Phys. J. C77, 406 (2017).

[39] M. Szydlowski, A. Stachowski, A. Borowiec, Eur. Phys. J. C77, 603 (2017).

[40] V.I. Afonso, G.J. Olmo, D. Rubiera-Garcia, Phys.Rev.D 97 (2018) 2, 021503.

[41] V.I. Afonso, G.J. Olmo, E. Orazi, D. Rubiera-Garcia, Eur.Phys.J.C 78 (2018) 10, 866.

[42] V.I. Afonso, G.J. Olmo, E. Orazi, D. Rubiera-Garcia, Phys.Rev.D 99 (2019) 4, 044040.

[43] A. Wojnar, Eur. Phys. J. C78 (2018) no.5, 421.

[44] A. Wojnar, Eur. Phys. J. C79 (2019) no.1, 51.

[45] A. Sergyeyev, A. Wojnar, Eur.Phys.J.C 80 (2020) 4, 313.

[46] A. Wojnar, Acta Phys.Polon.Supp. 13 (2020) 249.

[47] G.R. Caughlan, W. A. Fowler, At. Data Nucl. Data Tables, 40, 283 (1998).

[48] G. Raimann, Z. Phys. A Hadrons and Nuclei 347.1 (1993): 73-74.

[49] K. Masuda, H. Kawahara, D.W. Latham, A. Bieryla, M. Kunitomo, M. MacLeod, W. Aoki, AJ, Let., Vol. 881, No.1 (2019).

[50] G. Laughlin, P. Bodenheimer, F.C. Adams, AJ, 482, 420432 (1997).

[51] A. Wojnar, arXiv:2012.13927.

[52] I.N. Reid, The Astrophysical Journal 111, p. 2000 (1996).

[53] A.C Davis et al, Physical Review D 85.12 (2012): 123006. 